\title{
Interaction of Thiamine Deficiency and Voluntary Alcohol Consumption Disrupts Rat Corpus Callosum Ultrastructure
}

\author{
Xiaohua He ${ }^{1,2}$, Edith V Sullivan ${ }^{3}$, Roger K Stankovic', Clive G Harper*,' and Adolf Pfefferbaum ${ }^{3,4}$ \\ 'Department of Pathology (D06), University of Sydney, Sydney, NSW, Australia; '2Department of Pathophysiology, Medical School, Wuhan \\ University, Wuhan, PR China; ${ }^{3}$ Department of Psychiatry and Behavioral Sciences, Stanford University School of Medicine, Stanford, CA, USA; \\ ${ }^{4}$ Neuroscience Program, SRI International, Menlo Park, CA, USA
}

\begin{abstract}
The relative roles of alcohol and thiamine deficiency in causing brain damage remain controversial in alcoholics without the WernickeKorsakoff syndrome. Experimental control over alcohol consumption and diet are impossible in humans but can be accomplished in animal models. This experiment was designed to differentiate the separate and combined effects on the macro- and ultrastructure of the corpus callosum of thiamine deficiency and voluntary alcohol consumption. Adult male alcohol-preferring $(P)$ rats $(9$ chronically alcoholexposed and 9 water controls) received a thiamine-deficient diet for 2 weeks. There were four groups: five rats previously exposed to alcohol were treated with pyrithiamine (a thiamine phosphorylation inhibitor); five rats never exposed to alcohol were treated with pyrithiamine; four alcohol-exposed rats were treated with thiamine; and four rats never exposed to alcohol were treated with thiamine. On day 14 , thiamine was restored in all 18 rats; 2 weeks later the 10 pyrithiamine-treated rats received intraperitoneal thiamine. The rats were perfused 61 days post-pyrithiamine treatment at age 598 days. Brains were dissected and weight and volumes were calculated. Sagittal sections were stained to measure white matter structures. The corpus callosum was examined using transmission electron microscopy to determine density of myelinated fibers, fiber diameter, and myelin thickness. The corpus callosum in the alcohol/ pyrithiamine group was significantly thinner, had greater fiber density, higher percentage of small fibers, and myelin thinning than in the alcohol/thiamine and water/thiamine groups. Several measures showed a graded effect, where the alcohol/pyrithiamine group had greater pathology than the water/pyrithiamine group, which had greater pathology than the two thiamine-replete groups. Across all 16 rats, thinner myelin sheaths correlated with higher percentage of small fibers. Myelin thickness and axon diameter together accounted for $71 \%$ of the variance associated with percentage of small fibers. Significant abnormalities in the alcohol/pyrithiamine group and lack of abnormality in the alcohol-exposed/thiamine-replete group indicate that thiamine deficiency caused white matter damage. The graded abnormalities across the dually to singly treated animals support a compounding effect of alcohol exposure and thiamine depletion, and indicate the potential for interaction between alcohol and thiamine deficiency in human alcohol-related brain damage. Neuropsychopharmacology (2007) 32, 2207-22 I6; doi:I0.1038/sj.npp. I 30I332; published online I4 February 2007
\end{abstract}

Keywords: electron microscopy; alcohol; thiamine deficiency; alcohol-preferring $(P)$ rat; $M R I$; brain white matter

\section{INTRODUCTION}

Excessive drinking can lead to impairment of cognitive and motor function and structural brain changes-some permanent and some reversible. Functions commonly affected involve abstract problem solving, visuospatial and verbal learning, selective memory processes, perceptual motor skills, and postural stability (Butters and Granholm, 1987; Fein et al, 1990; Nixon et al, 2002; Oscar-Berman, 2000; Sullivan et al, 2000a, b, 2002). Disruption of frontopontocerebellar and cerebellothalamocortical circuitry may underlie the mainstay of these neuropsychological deficits

*Correspondence: Dr CG Harper, Department of Pathology (D06), University of Sydney, Sydney, NSW 2006, Australia, Tel: +61 293513663, Fax: + 61293513429, E-mail: cliveh@med.usyd.edu.au Received 22 August 2006; revised 20 October 2006; accepted 17 November 2006
(Sullivan, 2003; Sullivan and Pfefferbaum, 2005). Hence, a potential substrate of dysfunction may arise from structural changes in the white matter or 'hard wiring' of the brain, composed largely of myelinated axons. Pathological changes in white matter have been described in human in vivo (Pfefferbaum et al, 1992) and autopsy (Harper and Kril, 1991; Harper and Kril, 1994) studies, and in animal models (Hansen et al, 1991; Langlais and Zhang, 1997). Alcoholics typically have abnormally low brain weight and volume, which in postmortem analysis is largely accounted for by white matter volume shrinkage relative to nonalcoholic control cases (Harper, 1998b).

Specific white matter structures of the brain, like the corpus callosum, are particularly affected by alcoholism (Harper and Kril, 1988; Hommer et al, 1996; Pfefferbaum et al, 1996) and therefore useful in the study of its progression. The precise structural changes underlying the white matter volume loss, restoration with alcohol 
abstinence, and disruption of microstructural integrity remains unclear but probably involves both myelination and axonal integrity, inferred from in vivo MR diffusion tensor imaging studies (Pfefferbaum et al, 2000, 2006c, d; Pfefferbaum and Sullivan, 2002, 2005), and may explain why tissue volume recovery appears incomplete with abstinence. Alcoholic brain pathology may have two components, one reflecting permanent change and one a transient change (Carlen et al, 1984). Regarding permanent effects, alcoholrelated neuronal loss has been documented in specific regions of the cerebral cortex (superior frontal association cortex), hypothalamus and cerebellum (Harper, 1998b). Such loss can result in axonal (Wallerian) degeneration and a permanent reduction in white matter volume. Structural changes in myelin, however, could explain the reversible white matter shrinkage that has been documented with serial magnetic resonance imaging (MRI) studies, following periods of abstinence from alcohol (Gazdzinski et al, 2005; O'Neill et al, 2001; Pfefferbaum et al, 1995; Shear et al, 1994). In support of this hypothesis, molecular studies of human brains have shown that the expression of genes encoding myelin proteins (Lewohl et al, 2000; Mayfield et al, 2002) and actual levels of myelin-associated proteins are low in alcoholic relative to control cases (Lewohl et al, 2005; Liu et al, 2006).

Mechanisms thought to cause such disruption in brain structural integrity include the toxic effects of ethanol (alcohol) itself or its metabolites. Supporting this position, a pathological study (Kril et al, 1997) found a relationship between reported maximum daily alcohol consumption and amount of white matter volume loss. However, many studies implicate thiamine deficiency as responsible for the pathology. Thiamine deficiency causes the WernickeKorsakoff syndrome (WKS), a devastating neurological disorder characterized by oculomotor signs, ataxia, and mental symptoms (Harper, 2006). WKS occurs in humans, typically chronic alcoholics (Harper, 1983), and can be replicated in animal models using pyrithamine alone (Langlais and Savage, 1995) or in combination with alcohol (Zimitat et al, 1990) as used in this study. The frequent occurrence of thiamine deficiency with chronic alcoholism makes it difficult to determine the exact cause of white matter lesions in alcoholics (Harper and Kril, 1990). The reason that alcoholics are prone to develop thiamine deficiency relates to decreased oral intake of food and the direct effects of alcohol on intestinal transport of thiamine (Gastaldi et al, 1989). The resulting reduced thiamine stores and impaired thiamine utilization impair normal central nervous system function (Thomson et al, 2002). Phillips et al (1987) have speculated that the withdrawal effects of highly intoxicating levels of alcohol contribute to the disruption of white matter integrity. Animal studies show significant shrinkage of the corpus callosum several months after only a single bout of pyrithiamine-induced thiamine deficiency (Langlais and Savage, 1995). Ultrastructural studies show splitting of myelin sheaths and swelling of periaxonal spaces within the cerebral cortex of pyrithiamine-treated rats (Takahashi et al, 1988). Definitive pathology that could account for reversible brain shrinkage, however, remains unidentified, indicating the need for further investigation of the potential interaction between thiamine deficiency and chronic alcohol exposure in white matter pathology. Because control over alcohol consumption and diet is not experimentally viable in humans, animal models are required. Accordingly, this study was designed to differentiate the separate and combined effects of thiamine deficiency and alcohol exposure in rats selectively bred to voluntarily consume alcohol (Li et al, 1979).

The present postmortem analysis used electron microscopy in the rats previously examined longitudinally with MRI before (Sullivan et al, 2006) and after (Pfefferbaum et al, 2006b) alcohol exposure and thiamine depletion and repletion (Pfefferbaum et al, 2006a). In vivo measurements of the corpus callosum indicated $36 \%$ growth over 12 months without alcohol exposure and attenuation of growth with substantial voluntary alcohol consumption and adequate nutrition, and further arrested growth with thiamine depletion. Here, we tested the following competing hypotheses: if alcohol exposure and thiamine deprivation had a compounding adverse effect, we would observe a graded pattern, whereby animals, which had experienced the combined alcohol exposure plus thiamine depletion by diet and intraperitoneal (i.p.) pyrithiamine, would show the greatest evidence for white matter fiber degeneration, whereas rats with either alcohol exposure or thiamine depletion alone would have more modest evidence of white matter disruption. Alternatively, if either alcohol exposure or thiamine deficiency were the sole cause of fiber pathology, then the dual exposure would not compound the pathology.

\section{MATERIALS AND METHODS}

\section{Animals}

All animals were alcohol-preferring $(\mathrm{P})$ rats, obtained from the breeding colony at the Indiana University Medical Center (Indianapolis, IN; courtesy of R Bell, $\mathrm{PhD}$ ). The rats were from an outbred Wistar foundational stock, which has undergone over 50 generations of selection for a preference $10 \%(\mathrm{v} / \mathrm{v})$ alcohol over concurrently available water. They were all anatomically normal as shown from their baseline MRI, conducted at the outset of the in vivo experiment and before alcohol or pyrithiamine treatment. The rats were singly housed in plastic tubs with water and food (vitamin and mineral enriched Teklad mouse and rat diet \#7001; Madison, WI) freely available in the home tubs at all times. The four study groups comprised a cohort of 18 adult male $\mathrm{P}$ rats, nine of which had chronic, intermittent exposure to alcohol under four schedules over the year before the present study (Pfefferbaum et al, 2006b); the remaining nine rats were controls, which had never received alcohol (Sullivan et al, 2006). At the start of the pyrithiamine i.p./ thiamine deficiency experiment, the rats were 452 days old, and they did not differ significantly in body weight or brain volume. Further, the two groups with prior alcohol exposure did not differ in the total amount of alcohol consumed across their one year of access.

\section{Thiamine Deprivation Treatment}

Following the protocol employed in earlier studies (Langlais and Zhang, 1997; Savage et al, 2000), all rats received thiamine-deficient chow (Pfefferbaum et al, 2006b). Ten rats 
(five alcohol-exposed and five nonexposed) received daily i.p. injections of pyrithiamine hydrobromide $(0.25 \mathrm{mg} / \mathrm{kg}$; Aldrich Chemicals, Castle Hill, NSW, Australia). The remaining four rats from each of the control and alcoholexposed groups received daily i.p. injections of thiamine $(0.40 \mathrm{mg} / \mathrm{kg})$. The treatment protocol was carried out for 14 consecutive days. At the end of the thiamine deprivation period, regular thiamine-enriched chow was resumed for all animals (vitamin and mineral enriched Teklad mouse and rat diet \#7001; Madison, WI), and 2 weeks later the 10 pyrithiamine-treated animals were administered $100 \mathrm{mg} / \mathrm{kg}$ thiamine i.p. The full protocol and in vivo outcome measures have been reported (Pfefferbaum et al, 2006a).

\section{Histological Procedures}

Of the 18 rats in the in vivo studies, two rats in the pyrithiamine/alcohol condition died prematurely (31 and 39 days post-pyrithiamine treatment, 30 and 22 days before the planned perfusion time) despite vigorous efforts with gavage and dietary supplementation for recovery. Although the brain of the rat which died 39 days post-pyrithiamine treatment was available for histological study, its data were not included in the group data because of lack of control over postmortem brain extraction time and conditions and the substantially shorter time that rat relative to the rats that survived had for recovery.

At 61 days post-pyrithiamine treatment at age 598 days, the 16 animals that survived the full length of the experiment were anaesthesized and perfused through the ascending aorta with a fixative solution containing $4 \%$ paraformaldehyde in $0.1 \mathrm{M}$ phosphate buffer. The brains were removed after sectioning the brain stem. Brain weights were obtained and volumes calculated using a fluid displacement method (Zimitat et al, 1990). The brains were then sectioned sagittally in the midline to separate the cerebral and cerebellar hemispheres and divide the brain stem. The left hemisphere was photographed and then embedded in paraffin medial face down. Sagittal sections were cut and stained with hematoxylin and eosin and luxol fast blue, the latter to highlight the white matter for measurement of the area and thickness of the corpus callosum. The cerebellum and brain stem were separated from the right cerebral hemisphere by a horizontal cut at the level of the upper midbrain. The right hemisphere was cut in the coronal plane at approximately $1.5 \mathrm{~mm}$ intervals and samples of the genu, body, and splenium of the corpus callosum were taken for transmission electron microscopic examination. Blocks $(1 \mathrm{~mm} \times 2 \mathrm{~mm} \times 1 \mathrm{~mm})$ were dehydrated, osmicated, and embedded in plastic. A series of semi-thin sections $(1 \mu \mathrm{m})$ were taken from each block, mounted on copper grids and stained (uranyl acetate for $15 \mathrm{~min}$ and lead citrate for $5 \mathrm{~min}$ ) and examined on a transmission electron microscope (Zeiss 902, North Ryde, NSW, Australia). Digital transmission electron microscopic photographs $(\times 4400)$ were taken in a random pattern to determine the microscopic structure of the corpus callosum.

\section{Morphometric Analysis}

The area and thickness of the corpus callosum were measured using digital photographs of the luxol fast stained sections of the medial sagittal surfaces of the rat brains, using a semi-automated image analysis system (ImagePro Plus V. 4.5, CyberneticsMedia, Silver Spring, MD, USA). Measurements were made six times for each rat and means calculated. The digital transmission electron microscopic photographs were used for the measurement of myelineated fiber numbers and diameter, axon diameter and myelin sheath thickness using ImagePro Plus V. 4.5 (CyberneticsMedia, USA). Approximately 150 fibers per tissue block were selected for detailed morphometric analysis. The density of myelinated fibers, percent of small fibers, and $g$ ratio (axon diameter/total nerve fiber diameter) were determined. Cases were coded to facilitate blind quantification. All dimensions were corrected against standard measurement bars.

\section{Statistical Analyses}

Group differences for pyrithiamine $v s$ thiamine and alcohol $v s$ no alcohol exposure were tested with one- and two-way analysis of variance (ANOVA). Follow-up comparisons between pairs of groups were subjected to $t$-tests. Hypothesized differences were considered significant with $\alpha=0.05$ (one-tailed $p$-values for paired comparisons). Pearson correlations and multiple regression analysis examined relations between and among variables. Because of the small sample sizes, the parametric analyses were confirmed with nonparametric tests.

\section{RESULTS}

\section{Brain Weight and Brain Volume}

Although the volumes in the pyrithiamine groups were lower than the volumes in the thiamine-replete groups (Figure 1), the differences among the four groups were not significant for either brain weight $(F(3,15)=0.272$, $p=0.84)$ or brain volume $(\mathrm{F}(3,15)=0.803, p=0.52)$.

\section{Corpus Callosum Measurements}

Examples of TEM plates are presented in Figure 2. Mean$\mathrm{s} \pm \mathrm{SEM}$ of each quantified measure of the corpus callosum are presented in Figure 3, and results of the statistical comparisons are described next.

Area, thickness, and fiber density. One-way ANOVAs revealed significant group effects for the area $(\mathrm{F}(3,15)=3.94, p=0.0361)$ and thickness of the corpus

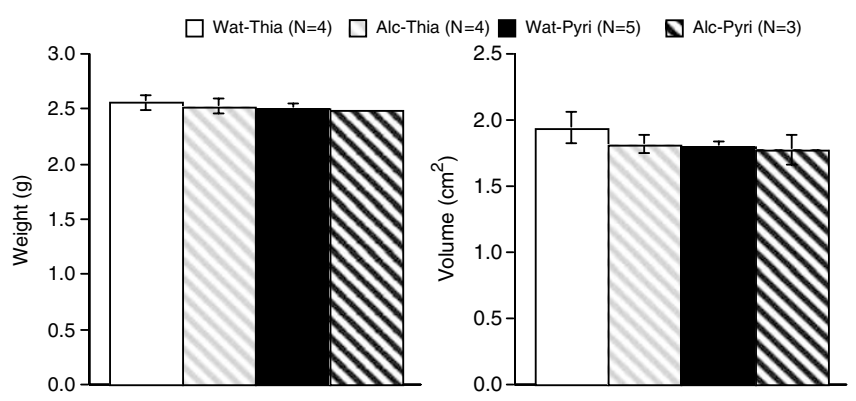

Figure I Mean \pm SEM of brain weight and volume of each of the four groups of rats. 

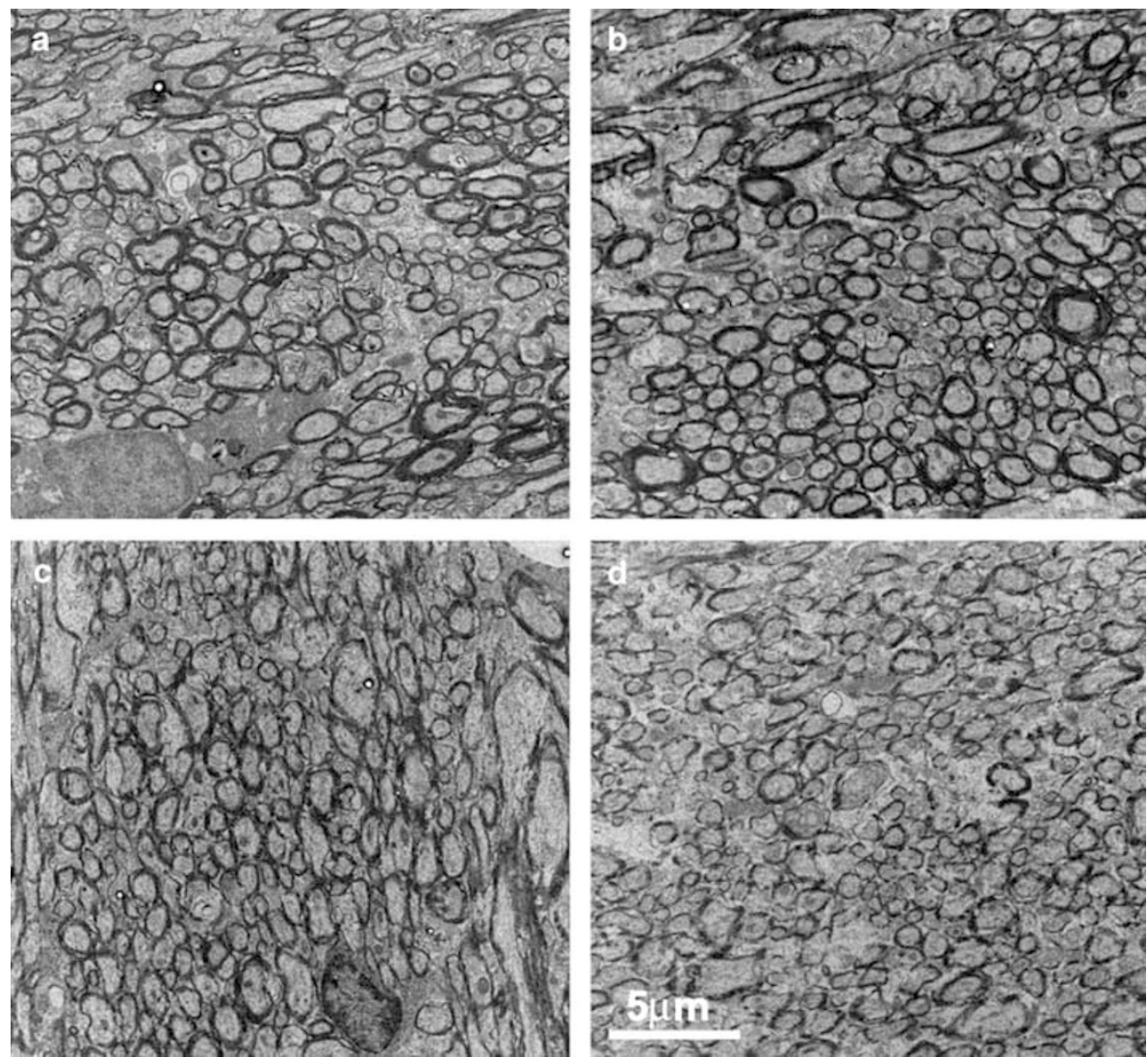

Figure 2 Transmission electron micrographs of sagittal sections of the corpus callosum: (a) water/thiamine, (b) alcohol/thiamine, (c) water/pyrithiamine, (d) alcohol/pyrithiamine. The animals in the alcohol/pyrithiamine group (d) had significantly more smaller fibers, and the fiber diameter and myelin sheath were significantly thinner compared with the remaining three groups. Scale bar is $5 \mu \mathrm{m}$.

callosum $(\mathrm{F}(3,15)=9.252, p=0.0019)$. Paired group comparisons indicated graded effects for the thickness measures, where the alcohol/pyrithiamine group had thinner corpora callosa than the water/pyrithiamine group $(t(6)=3.329, p=0.0079)$, which in turn had smaller callosal measures than did either thiamine group (water $t(7)=4.549, p=0.0013$; alcohol $t(7)=2.635, p=0.0168)$. The callosal area of the alcohol/pyrithiamine group was smaller than the alcohol/thiamine group $(t(5)=3.139$, $p=0.0129$ ). Two-way ANOVA also supported the graded effect with alcohol/water $v s$ pyrithiamine/thiamine interactions for callosal thickness $(\mathrm{F}(1,12)=6.84, p=0.0226)$ and a trend for callosal area $(\mathrm{F}(1,12)=4.073, p=0.0665)$. Oneway ANOVAs revealed a trend for density of the myelinated nerve fibers $(F(3,15)=3.314, p=0.0571)$, where the alcohol/pyrithiamine group had greater fiber density than the remaining three groups (comparison with water/thiamine $t(5)=2.265, p=0.0364$; alcohol/thiamine $t(5)=1.975, p=$ 0.0526 ; water/pyrithiamine $t(6)=1.975, p=0.0478)$.

Percent of small fibers. We categorized small fibers as those with a diameter less than $0.65 \mu \mathrm{m}$. One-way ANOVA $(\mathrm{F}(3,15)=7.135, p=0.0052)$ and follow-up tests supported the hypothesized graded effect (Figure 2). Specifically, the alcohol/pyrithiamine animals tended to have a higher percent of small myelinated fibers than the water/pyrithiamine group $(t(6)=1.659, p=0.0751)$, which in turn had a higher percentage of small fibers than the alcohol/thiamine group $(t(7)=2.365, p=0.0249)$ and tended to have a higher percentage than the water/thiamine group $(t(7)=1.814$, $p=0.0563)$. The two thiamine groups did not differ significantly from each other $(t(6)=1.23, p=0.13)$. This pattern of differences was also noted with a two-way ANOVA (thiamine/pyrithiamine $v s$ water/alcohol), which revealed a trend for an interaction $(\mathrm{F}(1,12)=4.249$, $p=0.0616)$, suggesting that the pyrithiamine-treated animals with alcohol exposure had a disproportionately greater percentage of small fibers than did the thiamine-treated groups.

Myelin sheath thickness. In general, one-way ANOVA indicated that the mean myelin sheaths of the pyrithiamine groups were thinner than those in the thiamine groups $(\mathrm{F}(3,15)=6.13, p=0.009)$. Follow-up tests revealed that the alcohol/pyrithiamine group had a thinner myelin sheath than either the water/thiamine group $(t(5)=5.681$, $p=0.0012)$ or the alcohol/thiamine group $(t(5)=3.545$, $p=0.0082)$. The water/pyrithiamine group had significantly thinner myelin sheaths than the water/thiamine $(t(7)=2.342, p=0.0259)$ but the difference from the alcohol/thiamine group was only a trend $(t(7)=1.666$, $p=0.0698$ ).

Axon diameter. One-way ANOVA yielded a trend toward an overall group difference in axon diameter $(\mathrm{F}(3,15)=2.108, p=0.1526)$. The greatest differences were 
Wat-Thia $(\mathrm{N}=4) \quad$ Wat-Pyri $(\mathrm{N}=5)$

Alc-Thia $(\mathrm{N}=4) \quad \mathbf{\nabla}$ Alc-Pyri $(\mathrm{N}=3)$
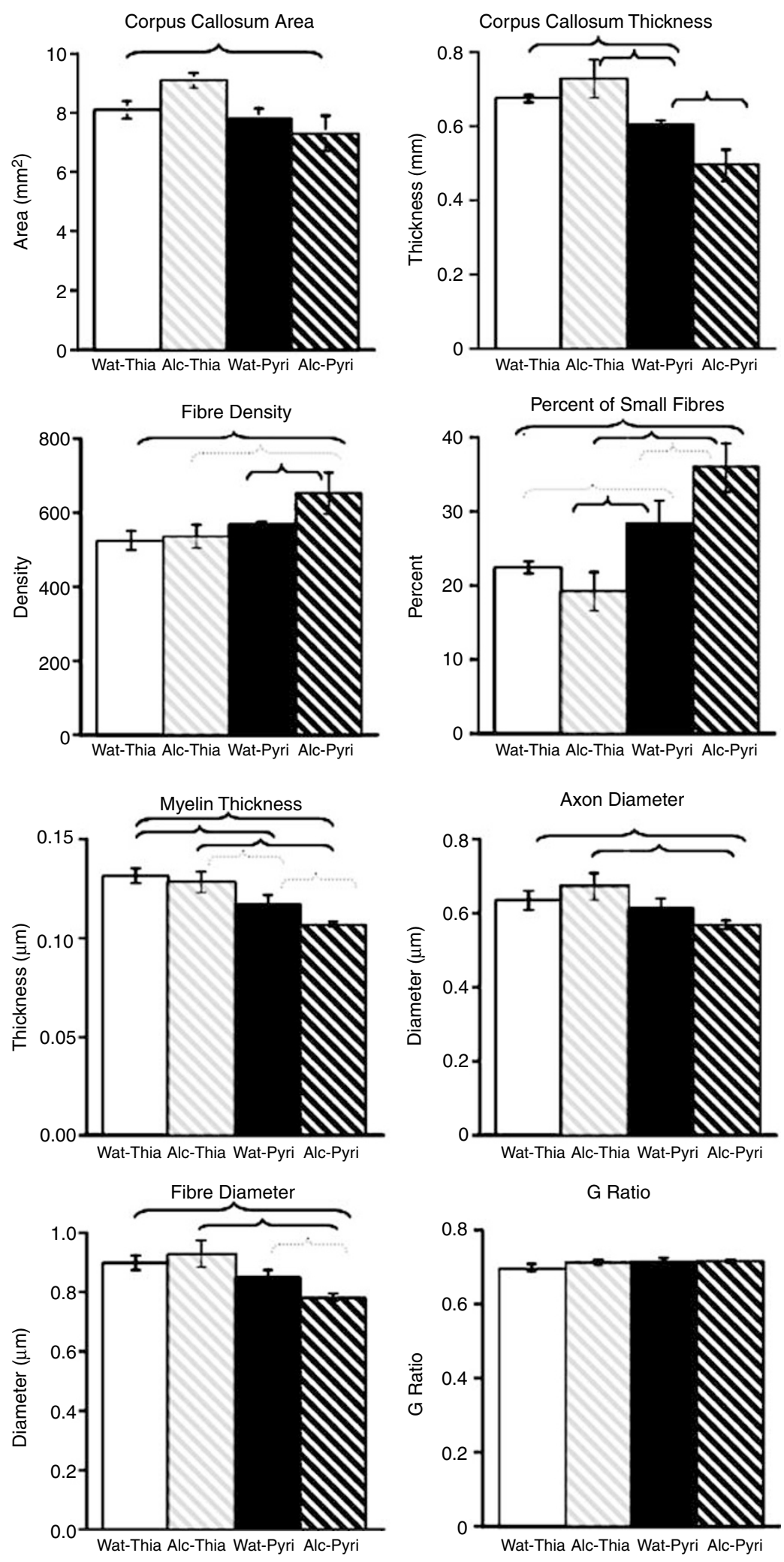

Figure 3 Mean \pm SEM of macrostructural and ultrastructural measures of the corpus callosum for the four groups (water/thiamine, alcohol/thiamine, water/pyrithiamine, and alcohol/pyrithiamine). The solid braces indicate group differences significant on one-tailed testing; dashed braces indicate statistical trends. 
related to the thin axon diameter of the alcohol/pyrithiamine group relative to the water/thiamine group $(t(5)=2.131, p=0.0432)$ and the alcohol/thiamine group $(t(5)=2.38, p=0.0316)$.

Fiber diameter. Group differences by one-way ANOVA were significant for total fiber diameter $(\mathrm{F}(3,15)=3.785$, $p=0.0403)$. The mean total fiber diameter in the alcohol/ pyrithiamine group was smaller than those of the remaining three groups (water/thiamine $t(5)=3.676, p=0.0072$; alcohol/thiamine $t(5)=2.793, p=0.0191$; water/pyrithiamine $t(6)=1.881, p=0.0545)$.

$\mathrm{G}$ ratio (axon diameter/total nerve fiber diameter). There were no significant differences in the $G$ ratio among the four groups $(\mathrm{F}(3,15)=0.722, p=0.558)$.

\section{Relations Among Callosal Measures}

Across all 16 rats myelin thickness correlated with axon diameter $(r=0.53, p=0.0363)$ (Figure 4). Within the combined pyrithiamine-treated groups $(N=8)$, percent of small myelinated fibers was related to fiber diameter $(r=-0.82, p=0.0131)$ and axon diameter $(r=-0.72$, $p=0.0461)$, and modestly to myelin thickness $(r=-0.63$, $p=0.0954)$. Multiple regression analysis using myelin thickness and axon diameter to predict percent of small fibers revealed that together these variables accounted for $71 \%$ of the variance and that axon diameter $(t=2.318$, $p=0.0682$ ) made a greater independent contribution to the variance than did myelin thickness $(t=1.835, p=0.1259)$.

\section{Relations Between Antemortem and Postmortem Measures}

All rats had undergone MRI scanning antemortem. Analysis based on all 16 rats revealed significant positive correlations between the length of the corpus callosum measured on

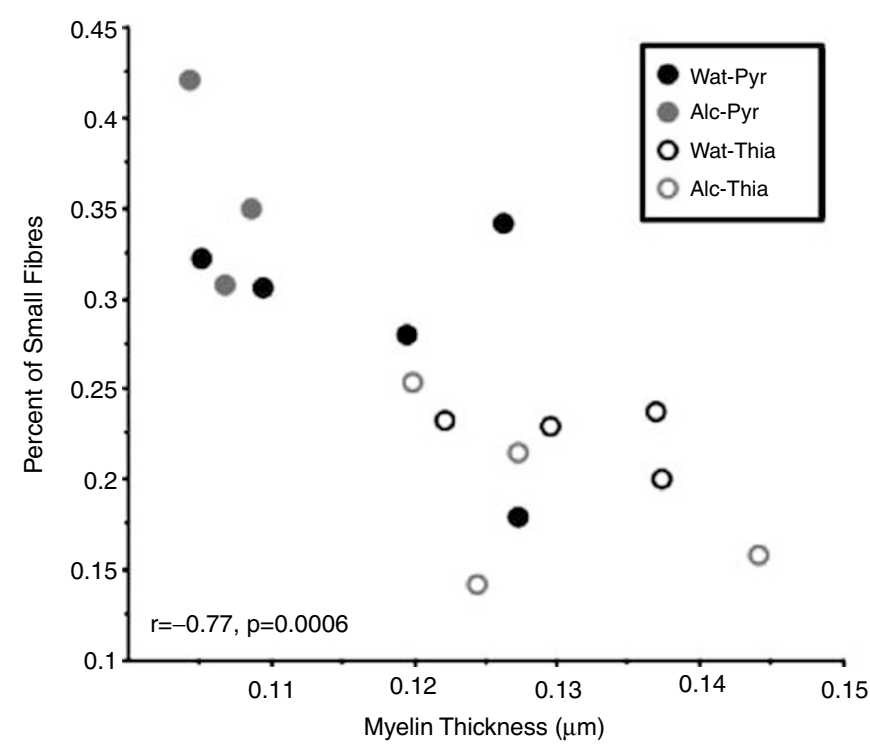

Figure 4 Correlations between myelin thickness and percentage of small fibers in the 16 rats color and symbol coded by treatment group.
MRI and brain weight $(r=0.75, p=0.0008$; Rho $=0.73$, $p=0047)$, and brain volume $(r=0.53, p=0.0336$; Rho $=$ $0.45, p=0.0796)$ measured postmortem.

\section{Histological Features of the Alcohol/Pyrithiamine Rat Excluded from Group Analysis}

One rat in the alcohol/pyrithiamine group died 22 days before the other animals but its brain was available for postmortem examination. Analysis indicated enlarged callosal area and thickness, low $G$ ratio, reduction in the density of myelinated nerve fibers, and greater space between the fibers. It is possible this rat died during the sub-acute phase of the experiment, whereas the other animals were killed during the early recovery stages.

\section{DISCUSSION}

The objective of this postmortem analysis using alcohol preferring $(\mathrm{P})$ Wistar rats was to examine the separate and combined effects of voluntary alcohol consumption and thiamine deficiency (pyrithamine treatment). The aim was to develop a better understanding of the mechanisms of brain damage in alcohol-related disorders including the WKS. The focus was on white matter damage, particularly the macrostructure and ultrastructure of the corpus callosum. Antemortem, the animals were divided into four groups: rats previously exposed to alcohol and later treated with pyrithiamine; rats never exposed to alcohol and then treated with pyrithiamine; alcohol-exposed rats treated with thiamine; rats never exposed to alcohol and treated with thiamine. Although the groups did not differ in brain weight or volume, quantitative morphometric analysis of the structure of the corpus callosum revealed smaller callosal area and thickness in rats with the combined alcohol and thiamine deficient history compared with rats that drank alcohol or were only thiamine deficient. The apparent paradox between the MRI and pathological data is explained by the fact that the group differences in brain weight and brain volume, while not statistically significantly different, were graded downward in a predictable way. That is, the water/thiamine group (controls) had the greatest weight and largest volume, and the alcohol/pyrithiamine group had the smallest of both measures postmortem, even though the overall differences were not significant. Nonetheless, when values of each of these measures, graded by group, were related to values from in vivo corpus callosum length, which showed the same graded pattern as the postmortem measures, the resulting correlations were also stepwise, where rats with shorter corpora callosa had smaller brain volumes and weights, whereas rats with longer callosa had larger volumes and weights.

Ultrastructural studies of the corpus callosum showed that the total myelinated fiber diameter was smaller and the myelin sheath was thinner in the alcohol/pyrithiamine group compared with the alcohol/thiamine-replete group, suggesting that thiamine deficiency alone was sufficient to cause white matter degeneration. However, history of chronic alcohol consumption, even though inadequate to cause substantial damage on its own, exacerbated the thiamine deficiency neuropathology as evidenced by a 
compounding or graded effect. Specifically, the alcohol/ pyrithiamine group had the most severe pathology of all four groups in callosal thickness, fiber density, fiber diameter, and myelin thickness. Further evidence for the poor condition of the dually treated rats was that the only two rats that failed to survive the full study course, and thus were not included in the current postmortem analysis, were in the alcohol/pyrithiamine group.

These findings are consistent with the in vivo complement of this study using MRI and MR spectroscopy (MRS). In vivo these rats showed significant changes in the early stages of pyrithiamine treatment followed by partial recovery with thiamine repletion. The pyrithiamine rats with prior alcohol exposure exhibited attenuated recovery in the thalamus in terms of structure and a marker of neuronal viability, $N$-acetylaspartate, and arrested growth of the corpus callosum, compared with other rats (Pfefferbaum et al, 2006a). In a similar postmortem study in rats using the pyrithiamine model (but not MRI or MRS), severe thiamine deficiency resulted in shrinkage of the corpus callosum (Langlais and Zhang, 1997). The shrinkage persisted for several months after recovery as did associated cognitive deficits. Other studies have shown that some of the earliest structural changes observed in thiamine-deficient rats and mice is intracellular edema of oligodendrocytes (the CNS cells that form myelin) and myelin (Robertson et al, 1968; Watanabe and Kanabe, 1978).

\section{White Matter Constituents and Morphology}

Smaller myelinated fiber diameter was accompanied by thinner myelin sheaths in the thiamine-deficient animals. This co-occurrence has not been previously described with respect to thiamine deficiency or alcohol toxicity, except in a fetal alcohol model. Phillips et al (1991) showed that myelin thickness as a function of axon diameter was decreased in alcohol-exposed rats from 10 to 90 days, indicating a permanent reduction in the relative thickness of myelin sheaths. After maternal alcohol administration the offspring also exhibited decreased expression of myelin basic protein (MBP) (Ozer and Halpain, 2000). The expression of MBP and myelin associated glycoprotein (MAG) was also affected by exposure to alcohol during postnatal development, especially during periods of rapid myelination (Zoeller et al, 1994). Direct exposure of cultures of differentiating CG-4 oligodendrocytes, the myelin forming cells of the CNS, to varying concentrations of alcohol alters the developmentally regulated expression of MBP (Bichenkov and Ellingson, 2002). Proteolipid protein (PLP) and MBP are the major myelin proteins in the CNS and comprise approximately 50 and $30 \%$ of total CNS myelin proteins, respectively. These proteins are required for the highly ordered and compact structure of myelin and are specifically involved in stabilization and compaction of the myelin sheath (Boison and Stoffel, 1994; Omlin et al, 1982). The myelin-related genes also influence biosynthesis (Schaeren-Wiemers et al, 1995). Hence, alteration of the gene expression and amounts of myelin-related proteins are likely to alter the structure and function of the myelin sheath and ultimately the conduction of action potentials.

Recent molecular studies support the hypothesis that alcohol-dependent individuals are liable to incur structural changes in myelin. Using microarray studies, Lewohl et al (2000) analyzed gene expression in autopsy samples of human superior frontal cortex of patients with a history of alcoholism. This brain region is particularly affected by chronic alcoholism (Harper and Kril, 1990; Pfefferbaum et $a l, 1997)$, with pronounced change in myelin gene expression as well as changes in genes coding for a number of synaptic and signal transduction proteins. In a study by Mayfield et al (2002), similar changes were observed in the expression of MAG, apolipoprotein D, glial fibrillary acidic protein, oligodendrocyte myelin glycoprotein, and PLP. White matter changes have been shown to be more severe in alcoholics with than without WKS (Harper, 1998a). Alexander-Kaufman et al (2007) have shown that the expression levels of two thiamine-dependent proteins, transketolase and pyruvate dehydrogenase E1 $\beta$-subunit, were reduced in alcoholics who had no clinical or pathological evidence of WKS, suggesting subclinical impairment of thiamine metabolism in some alcoholics putting them at risk for WKS.

The density of myelinated nerve fibers was substantially higher and the axons were more densely packed in the alcohol/pyrithiamine group than the other groups. The greater fiber density may have been the result of fiber deletion, possibly from Wallerian degeneration, or alternatively, regeneration (Tom et al, 2004), likely limited to recovery of small fibers (Wiggins et al, 1985). Our observation of a greater percentage of small fibers in the dually treated rats is consistent with two competing hypotheses: regeneration of small fibers and selective shrinkage or deletion of large fibers. The preferred hypothesis is that it is a consequence of selective loss or shrinkage of large fibers rather than a gain in numbers of small myelinated fibers. Although regeneration of nerve fibers is known to occur in the peripheral nervous system, little information supports such a mechanism in the central nervous system (Kim et al, 2006). In the peripheral neuropathy caused by thiamine deficiency (both alcoholic and nonalcoholic), large myelinated fibers are affected more than small myelinated fibers (Koike et al, 2003). By contrast, these authors noted that in pure alcoholic neuropathy, small myelinated fibers seem to be more affected. An actual reduction of axon diameters has been observed to occur at an early stage of inflammatory demyelinating polyneuropathy (peripheral nervous system) before much destruction of the myelin sheath has occurred (Prineas and McLeod, 1976). The relationship between myelinated fiber size and neuronal type in the central nervous system is poorly understood, although it is generally agreed that there is a good correlation between axonal size and conduction velocity, which has functional implications (Lee et al, 1986).

Human alcoholism can result in cortical neuronal loss and Wallerian degeneration of axons. Using a quantitative morphometric technique to study myelinated nerve fibers in the subcortical white matter of human cases with uncomplicated alcoholism, however, Tang et al (2004) were unable to show degeneration of axons. Thus, alcohol per se may not be the main cause of white matter disruption. This possibility is in line with our findings that thiamine deficiency was the principal single pathogenic mechanism causing brain white matter damage. 
The molecular mechanisms responsible for thiamine deficiency-induced white matter damage are unclear. The damage may result from altered lipid synthesis and metabolism (Heroux and Butterworth, 1995; Pannunzio et al, 2000) or oxidative stress (Todd and Butterworth, 1999; Zou and Crews, 2006). Myelin has a very low concentration of the thiamine cofactor thiamine diphosphate and thus may be especially susceptible to reduced energy metabolism.

\section{Combined Alcohol/Thiamine}

Alcohol abuse in conjunction with thiamine deficiency has severe effects on brain damage compared with thiamine deficiency alone. Zimitat et al (1990) showed that the onset of the clinical stages of neurological disease related to thiamine deficiency occurred significantly earlier and progressed faster in thiamine-deficient rats that received alcohol than in those that did not. Phillips et al (1987) reported that, after a thiamine-deficient diet or ethanol vapor exposure, no significant cytotoxicity was observed in mouse cerebellum; however, when given a combined thiamine-deficient diet with ethanol, axon terminal degeneration was present in deep cerebellar nuclei. Recent in vitro research also shows that neither ethanol treatment nor withdrawal from exposure significantly increased cerebellar cytotoxicity, whereas treatment with pyrithiamine significantly increased cytotoxicity $21 \%$ above levels observed in control tissue. Furthermore, cultures treated with both ethanol and pyrithiamine displayed a marked increase in cytotoxicity (60-90\%) above levels observed in control cultures (Mulholland et al, 2005). These results suggest that the operation of different mechanisms that involve interactions between the metabolic effects of thiamine deficiency and a neurotoxic effect of alcohol (Ciccia and Langlais, 2000; Crowe and El-Hadj, 2002). Therefore, assessment of the pathological features of 'alcoholic brain damage' requires consideration of the direct toxicity of ethanol or its metabolites and the concomitant effects of thiamine deficiency.

Although the interpretation of these data is limited by the small sample size, a longitudinal study of this length, design, and multidisciplinary nature provides a novel approach for clarifying the role of thiamine deficiency in producing ultrastructural white matter damage and for the compounded effect from chronic and excessive alcohol consumption. Indeed, the combined in vivo and postmortem experiment requires replication with larger samples and graded thiamine depletion.

\section{CONCLUSION}

This experiment, although limited by small samples, revealed that white matter lesions were more evident in the alcohol/pyrithiaimine group than in the water/pyrithiamine group and supports the hypothesis that chronic voluntary alcohol consumption exacerbates thiamine deficiency and untoward effects on brain white matter. This compounded effect indicates the potential for an alcohol/ thiamine deficiency interaction in producing alcoholrelated brain damage in human alcoholism. Although thiamine deficiency is well established to be the principal underlying cause of Wernicke's encephalopathy and its sequela, Korsakoff's syndrome (Thomson, 2000; Victor et al, 1971), the role of nutritional deficiency remains undetermined in chronic 'uncomplicated' alcoholism, but postmortem study indicates that WE is underdiagnosed in life (Harper, 1979; Martin et al, 2003; Torvik, 1991). Uncomplicated alcoholics likely sustain repeated bouts of less profound nutritional deficiency, which may contribute to their milder, in vivo neuroradiological characteristics relative to those marking WKS (Sullivan, 2000). Further, genetic variation in thiamine metabolism and storage may result in differential vulnerability to $\mathrm{WE}$ and KS with exacerbation from concomitant alcoholism (Manzardo and Penick, 2006; Martin et al, 1993). These unsolved possibilities can be addressed with larger in vivo to postmortem animal studies that manipulate thiamine levels in the context of bouts of high alcohol exposure aimed at paralleling human alcoholism.

\section{ACKNOWLEDGEMENTS}

This research was supported by funds from NIAAA (AA05965 and AA13521-INIA granted to AP) and NHMRC. The authors would like to thank Dr Richard L Bell of the Indiana University Animal Genetics Facility for providing us with the selectively bred alcohol-preferring (P) rats, Dr Teresa Steininger and Mr Juan Orduna for performing the perfusion and fixation procedures, Dr Zhong An, Li for providing TEM photographs and Dr Chris Burke for help with histological procedures. The authors are also grateful to the Electron Microscope Unit at the University of Sydney.

\section{REFERENCES}

Alexander-Kaufman K, Cordwell S, Harper C, Matsumoto I (2007). A proteome analysis of the dorsolateral prefrontal cortex in human alcoholic patients. Proteomics (in press).

Bichenkov E, Ellingson JS (2002). Protein kinase C inhibitors counteract the ethanol effects on myelin basic protein expression in differentiating CG-4 oligodendrocytes. Brain Res Dev Brain Res 139: 29-38.

Boison D, Stoffel W (1994). Disruption of the compacted myelin sheath of axons of the central nervous system in proteolipid protein-deficient mice. Proc Natl Acad Sci USA 91: 11709-11713.

Butters N, Granholm E (1987). The continuity hypothesis: some conclusions and their implications for the etiology and neuropathology of alcoholic Korsakoff's syndrome. In: Parsons OA, Butters N, Nathan P (eds). Neuropsychology of Alcoholism: Implications for Diagnosis and Treatment. Guilford Press: New York. pp 176-206.

Carlen PL, Wilkinson DA, Wortzman G, Holgate R (1984). Partially reversible cerebral atrophy and functional improvement in recently abstinent alcoholics. Can J Neur Sci 11: 441-446.

Ciccia RM, Langlais PJ (2000). An examination of the synergistic interaction of ethanol and thiamine deficiency in the development of neurological signs and long-term cognitive and memory impairments. Alcoholism: Clin Exp Res 24: 622-634.

Crowe SF, El-Hadj D (2002). Phenytoin ameliorates the memory deficit induced in the young chick by ethanol toxicity in association with thiamine deficiency. Pharmacol Biochem Behav 71: 215-221.

Fein G, Bachman L, Fisher S, Davenport L (1990). Cognitive impairments in abstinent alcoholics. West J Med 152: 531-537. 
Gastaldi G, Casirola D, Ferrari G, Rindi G (1989). Effect of chronic ethanol administration on thiamine transport in microvillous vesicles of rat small intestine. Alcohol Alcohol 24: 83-89.

Gazdzinski S, Durazzo TC, Meyerhoff DJ (2005). Temporal dynamics and determinants of whole brain tissue volume changes during recovery from alcohol dependence. Drug Alcohol Depend 78: 263-273.

Hansen LA, Natelson BH, Lemere C, Niemann W, Deteresa R, Regan TJ et al (1991). Alcohol-induced brain changes in dogs. Arch Neurol 48: 939-942.

Harper C (1979). Wernicke's encephalopathy: a more common disease than realized. A neuropathological study of 51 cases. J Neurol, Neurosurg Psychiatry 42: 226-232.

Harper C (1998a). The neuropathology of alcohol-specific brain damage, or does alcohol damage the brain? J Neuropath Exp Neur 57: 101-110.

Harper C (1998b). The neuropathology of alcohol-specific brain damage, or does alcohol damage the brain? Neuropath Exp Neur 57: $101-110$.

Harper C (2006). Thiamine (vitamin B1) deficiency and associated brain damage is still common throughout the world and prevention is simple and safe Eu J Neurol 13: 1-6.

Harper C, Kril J (1991). If you drink your brain will shrink: neuropathological considerations. Alcohol and Alcohol 1(Suppl): 375-380.

Harper C, Kril J (1994). An introduction to alcohol-induced brain damage and its causes. Alcohol Alcohol 2(Suppl): 237-243.

Harper CG (1983). The incidence of Wernicke's encephalopathy in Australia - a neuropathological study of 131 cases. J Neurol Neurosurg Psychiatry 46: 593-598.

Harper CG, Kril JJ (1988). Corpus callosal thickness in alcoholics. $\mathrm{Br} J$ Addict 83: 577-580.

Harper CG, Kril JJ (1990). Neuropathology of alcoholism. Alcohol Alcohol 25: 207-216.

Heroux M, Butterworth RF (1995). Regional alterations of thiamine phosphate esters and of thiamine diphosphate-dependent enzymes in relation to function in experimental Wernicke's encephalopathy. Neurochem Res 20: 87-93.

Hommer D, Momenan R, Rawlings R, Ragan P, Williams W, Rio D et al (1996). Decreased corpus callosum size among alcoholic women. Arch Neurol 53: 359-363.

Kim J, Schafer J, Ming GL (2006). New directions in neuroregeneration. Expert Opin Biol Th 6: 735-738.

Koike H, Iijima M, Sugiura M, Mori K, Hattori N, Ito $\mathrm{H}$ et al (2003). Alcoholic neuropathy is clinicopathologically distinct from thiamine-deficiency neuropathy. Annals Neurol 54: 19-29.

Kril JJ, Halliday GM, Svoboda MD, Cartwright H (1997). The cerebral cortex is damaged in chronic alcoholics. Neuroscience 79: 983-998.

Langlais PJ, Savage LM (1995). Thiamine deficiency in rats produces cognitive and memory deficits on spatial tasks that correlate with tissue loss in diencephalon, cortex and white matter. Behav Brain Res 68: 75-89.

Langlais PJ, Zhang SX (1997). Cortical and subcortical white matter damage without Wernicke's encephalopathy after recovery from thiamine deficiency in the rat. Alcohol Clini Experimen Res 21: 434-443.

Lee KH, Chung K, Chung JM, Coggeshall RE (1986). Correlation of cell body size, axon size, and signal conduction velocity for individually labelled dorsal root ganglion cells in the cat. J Compar Neurol 243: 335-346.

Lewohl J, Wang L, Miles M, Zhang L, Dodd P, Harris R (2000). Gene expression in human alcoholism: microarray analysis of frontal cortex. 24: 1873-1882.

Lewohl JM, Wixey J, Harper CG, Dodd PR (2005). Expression of MBP, PLP, MAG, CNP, and GFAP in the Human Alcoholic Brain. Alcohol Clin Exp Res 29: 1698-1705.
Li TK, Lumeng L, McBride WJ, Waller MB (1979). Progress toward a voluntary oral consumption model of alcoholism. Drug Alcohol Depend 4: 45-60.

Liu J, Lewohl JM, Harris RA, Iyer VR, Dodd PR, Randall PK et al (2006). Patterns of gene expression in the frontal cortex discriminate alcoholic from nonalcoholic individuals. Neuropsychopharmacology 31: 1574-1582.

Manzardo AM, Penick EC (2006). A theoretical argument for inherited thiamine insensitivity as one possible biological cause of familial alcoholism. Alcohol: Clin Exp Res 30: 1545-1550.

Martin PR, McCool BA, Singleton CK (1993). Genetic sensitivity to thiamine deficiency and development of alcoholic organic brain disease. Alcohol: Clin Expe Res 17: 31-37.

Martin PR, Singleton CK, Hiller-Sturmhofel S (2003). The role of thiamine deficiency in alcoholic brain disease. Alcohol Res Health 27: 134-142.

Mayfield RD, Lewohl JM, Dodd PR, Herlihy A, Liu J, Harris RA (2002). Patterns of gene expression are altered in the frontal and motor cortices of human alcoholics. J Neurochemi 81: 802-813.

Mulholland PJ, Self RL, Stepanyan TD, Little HJ, Littleton JM, Prendergast MA (2005). Thiamine deficiency in the pathogenesis of chronic ethanol-associated cerebellar damage in vitro. Neuroscience 135: 1129-1139.

Nixon SJ, Tivis R, Ceballos N, Varner JL, Rohrbaugh J (2002). Neurophysiological efficiency in male and female alcoholics. Prog Neuro-Psychopharmacol Biol Psychiatry 26: 919-927.

O'Neill J, Cardenas VA, Meyerhoff DJ (2001). Effects of abstinence on the brain: quantitative magnetic resonance imaging and magnetic resonance spectroscopic imaging in chronic alcohol abuse. Alcohol: Clini Expl Res 25: 1673-1682.

Omlin FX, Webster HD, Palkovits CG, Cohen SR (1982). Immunocytochemical localization of basic protein in major dense line regions of central and peripheral myelin. J Cell Biol 95: $242-248$

Oscar-Berman M (2000). Neuropsychological vulnerabilities in chronic alcoholism. In: Noronha A, Eckardt M, Warren K (eds). Review of NIAAA's Neuroscience and Behavioral Research Portfolio, NIAAA Research Monograph No 34. National Institutes of Health: Bethesda, MD, USA. pp 437-472.

Ozer RS, Halpain S (2000). Phosphorylation-dependent localization of microtubule-associated protein MAP2c to the actin cytoskeleton. Mol Biol Cell 11: 3573-3587.

Pannunzio P, Hazell AS, Pannunzio M, Rao KV, Butterworth RF (2000). Thiamine deficiency results in metabolic acidosis and energy failure in cerebellar granule cells: an in vitro model for the study of cell death mechanisms in Wernicke's encephalopathy. J Neurosci Res 62: 286-292.

Pfefferbaum A, Adalsteinsson E, Bell RL, Sullivan EV (2006a). Development and resolution of brain lesions caused by pyrithiamine and dietary Induced thiamine deficiency and alcohol exposure in the alcohol-preferring (P) Rat: A longitudinal MR imaging and spectroscopy study. Neuropsychopharmacology Epub, May 24, 2006.

Pfefferbaum A, Adalsteinsson E, Sood R, Mayer D, Bell RL, McBride WJ et al (2006b). Part II: Longitudinal brain MRI study of the alcohol-preferring (P) rat: Effects of voluntary chronic alcohol consumption. Alcoh: Clini Exp Res 30: 1248-1261.

Pfefferbaum A, Adalsteinsson E, Sullivan EV (2006c). Dysmorphology and microstructural degradation of the corpus callosum: Interaction of age and alcoholism. Neurobiol Aging 27: 994-1009.

Pfefferbaum A, Adalsteinsson E, Sullivan EV (2006d). Supratentorial profile of white matter microstructural integrity in recovering alcoholic men and women. Biol Psychiatry 59: 364-372.

Pfefferbaum A, Lim KO, Desmond J, Sullivan EV (1996). Thinning of the corpus callosum in older alcoholic men: A magnetic resonance imaging study. Alcohol: Clini Exp Res 20: 752-757. 
Pfefferbaum A, Lim KO, Zipursky RB, Mathalon DH, Lane B, Ha CN et al (1992). Brain gray and white matter volume loss accelerates with aging in chronic alcoholics: a quantitative MRI study. Alcohol: Clini and Exp Res 16: 1078-1089.

Pfefferbaum A, Sullivan EV (2002). Microstructural but not macrostructural disruption of white matter in women with chronic alcoholism. Neuroimage 15: 708-718.

Pfefferbaum A, Sullivan EV (2005). Disruption of brain white matter microstructure by excessive intracellular and extracellular fluid in alcoholism: evidence from diffusion tensor imaging. Neuropsychopharmacology 30: 423-432.

Pfefferbaum A, Sullivan EV, Hedehus M, Adalsteinsson E, Lim KO, Moseley M (2000). In vivo detection and functional correlates of white matter microstructural disruption in chronic alcoholism. Alcohol: Clini Exp Res 24: 1214-1221.

Pfefferbaum A, Sullivan EV, Mathalon DH, Lim KO (1997). Frontal lobe volume loss observed with magnetic resonance imaging in older chronic alcoholics. Alcohol: Clini Exp Res 21: 521-529.

Pfefferbaum A, Sullivan EV, Mathalon DH, Shear PK, Rosenbloom MJ, Lim KO (1995). Longitudinal changes in magnetic resonance imaging brain volumes in abstinent and relapsed alcoholics. Alcohol: Clini Exp Res 19: 1177-1191.

Phillips DE, Krueger SK, Rydquist JE (1991). Short- and long-term effects of combined pre- and postnatal ethanol exposure (three trimester equivalency) on the development of myelin and axons in rat optic nerve. Int J Dev Neurosci 9: 631-647.

Phillips SC, Harper CG, Kril J (1987). A quantitative histological study of the cerebellar vermis in alcoholic patients. Brain 110: 301-314.

Prineas JW, McLeod JG (1976). Chronic relapsing polyneuritis. J Neurol Sci 27: 427-458.

Robertson DM, Wasan SM, Skinner DB (1968). Ultrastructural features of early brain stem lesions of thiamine-deficient rats. Am J Pathol 52: 1081-1097.

Savage LM, Candon PM, Hohmann HL (2000). Alcohol-induced brain pathology and behavioral dysfunction: using an animal model to examine sex differences. Alcohol Clini Exp Res 24: 465-475.

Schaeren-Wiemers N, Valenzuela DM, Frank M, Schwab ME (1995). Characterization of a rat gene, rMAL, encoding a protein with four hydrophobic domains in central and peripheral myelin. J Neurosci 15: 5753-5764.

Shear PK, Jernigan TL, Butters N (1994). Volumetric magnetic resonance imaging quantification of longitudinal brain changes in abstinent alcoholics. Alcohol: Clini Exp Res 18: 172-176.

Sullivan EV (2000). Human brain vulnerability to alcoholism: Evidence from neuroimaging studies. In: Noronha A, Eckardt M, Warren K (eds). Review of NIAAA's Neuroscience and Behavioral Research Portfolio, NIAAA Research Monograph No 34. National Institutes of Health: Bethesda, MD, USA. pp 473-508.

Sullivan EV (2003). Compromised pontocerebellar and cerebellothalamocortical systems: speculations on their contributions to cognitive and motor impairment in nonamnesic alcoholism. Alcohol: Clini Exp Res 27: 1409-1419.

Sullivan EV, Adalsteinsson E, Sood R, Mayer D, Bell RL, McBride WJ et al (2006). Part I: Longitudinal brain MRI brain study of the alcohol-preferring (P) rat: Adult brain growth. Alcohol: Clin Exp Res 30: 1234-1247.

Sullivan EV, Deshmukh A, Desmond JE, Lim KO, Pfefferbaum A (2000a). Cerebellar volume decline in normal aging, alcoholism, and Korsakoff's syndrome: Relation to ataxia. Neuropsychology 14: $341-352$.

Sullivan EV, Fama R, Rosenbloom MJ, Pfefferbaum A (2002). A profile of neuropsychological deficits in alcoholic women. Neuropsychology 16: 74-83.

Sullivan EV, Pfefferbaum A (2005). Neurocircuitry in alcoholism: A substrate of disruption and repair. Psychopharmacology (Berl) 180: $583-594$.

Sullivan EV, Rosenbloom MJ, Pfefferbaum A (2000b). Pattern of motor and cognitive deficits in detoxified alcoholic men. Alcohol: Clini Exp Res 24: 611-621.

Takahashi H, Nakazawa S, Yoshino Y, Shimura T (1988). Metabolic studies of the edematous cerebral cortex of the pyrithiaminetreated thiamine-deficient rat. Brain Res 441: 202-208.

Tang Y, Pakkenberg B, Nyengaard JR (2004). Myelinated nerve fibres in the subcortical white matter of cerebral hemispheres are preserved in alcoholic subjects. Brain Res 1029: 162-167.

Thomson AD (2000). Mechanisms of vitamin deficiency in chronic alcohol misusers and the development of the WernickeKorsakoff syndrome. Alcohol Alcohol 35(Suppl): 2-7.

Thomson AD, Cook CC, Touquet R, Henry JA (2002). The Royal College of Physicians report on alcohol: guidelines for managing Wernicke's encephalopathy in the accident and Emergency Department. Alcohol Alcohol 37: 513-521.

Todd K, Butterworth RF (1999). Mechanisms of selective neuronal cell death due to thiamine deficiency. Ann N Y Acad Sci 893: 404-411.

Tom VJ, Doller CM, Malouf AT, Silver J (2004). Astrocyteassociated fibronectin is critical for axonal regeneration in adult white matter. J Neurosci 24: 9282-9290.

Torvik A (1991). Wernicke's encephalopathy: Prevalence and clinical spectrum. Alcohol Alcohol 1(Suppl): 381-384.

Victor M, Adams RD, Collins GH (1971). The Wernicke-Korsakoff Syndrome. F.A. Davis Co: Philadelphia, PA.

Watanabe I, Kanabe S (1978). Early edematous lesion of pyrithiamine induced acute thiamine deficient encephalopathy in the mouse. J Neuropathol Exp Neurol 37: 401-413.

Wiggins RC, Bissell AC, Durham L, Samorajski T (1985). The corpus callosum during postnatal undernourishment and recovery: a morphometric analysis of myelin and axon relationships. Brain Res 328: 51-57.

Zimitat C, Kril J, Harper CG, Nixon PF (1990). Progression of neurological disease in thiamin-deficient rats is enhanced by Ethanol. Alcohol 7: 493-501.

Zoeller RT, Butnariu OV, Fletcher DL, Riley EP (1994). Limited postnatal ethanol exposure permanently alters the expression of mRNAS encoding myelin basic protein and myelin-associated glycoprotein in cerebellum. Alcohol Clin Exp Res 18: 909-916.

Zou J, Crews F (2006). CREB and NF-kappaB Transcription Factors Regulate Sensitivity to Excitotoxic and Oxidative Stress Induced Neuronal Cell Death. Cell Mol Neurobiol 26: 383-403. 